\title{
Microcirugía transoral láser en cáncer de laringe. Experiencia en el Complejo Asistencial Barros Luco. 2003-2009
}

\author{
Transoral Laser Microsurgery of Cancer of the Larynx. \\ Barros Luco Medical Center. Experience 2003-2009
}

Rodrigo Arregui V1', Esteban Ortúzar G', Rocco Calabrese¹.

\begin{abstract}
RESUMEN
Introducción: El cáncer de laringe es la segunda neoplasia maligna más frecuente en cabeza y cuello, existiendo varias alternativas terapéuticas para su manejo entre las que destaca el uso de láser para los estadios tempranos.

Objetivo: Evaluar los resultados oncológicos de los pacientes con diagnóstico de cáncer de laringe que se trataron mediante microcirugía transoral láser en el Hospital Barros Luco.

Material y método: Estudio retrospectivo de 30 pacientes operados en el Hospital Barros Luco desde el año 2003 al año 2009.

Resultados: Para los pacientes con tumor T1 se obtuvo una sobrevida global (ajustada), control local inicial y final de 100\%. En los pacientes T2 se observó una sobrevida global de $100 \%$ con un control local inicial y final de $81,8 \%$. Los pacientes $T 3$ también describieron una sobrevida global de $100 \%$ pero sin lograr control local inicial y final en ninguno de los pacientes.

Discusión: Los resultados de control oncológico obtenidos en nuestro Servicio para estadios tempranos son similares a lo descrito en la literatura.

Conclusiones: La cirugía transoral láser es un procedimiento seguro y con resultados oncológicos aceptables para el manejo de pacientes con patología neoplásica de laringe en estadios tempranos.
\end{abstract}

Palabras clave: Cirugía transoral láser, cáncer de laringe.

\section{ABSTRACT}

Introduction: Laryngeal cancer is the second most common malignancy in head and neck, and there are several treatment options for their management, wherein the use of lasers for early stages.

Aim: To evaluate the oncological results of patients with a diagnosis of laringeal cancer, they were treated by transoral laser microsurgery at Hospital Barros Luco.

Material and method: Retrospective study of 30 medical records of patients operated at the Barros Luco Hospital from 2003 to 2009.

Results: For patients with T1 tumor overall survival (adjusted) initial and final local control of $100 \%$ was obtained. In T2 patients overall survival was 100\% with an initial

${ }^{1}$ Médico, Servicio de Salud Metropolitano Sur. Hospital Barros Luco Trudeau. 
and final local control of $81.8 \%$. Patients T3 described a $100 \%$ overall survival but without achieving local control and final control in none of the patients.

Discussion: Cancer control results obtained in our service for early stages are similar to those described in the literature.

Conclusions: Transoral laser surgery is a safe procedure with acceptable oncological outcomes in management of patients with neoplastic disease of the larynx in early stages.

Key words: Transoral laser surgery, cancer of the larynx.

\section{INTRODUCCIÓN}

El cáncer de laringe se encuentra en el segundo lugar de prevalencia dentro de las neoplasias malignas de cabeza y cuello a nivel mundial, estimándose 11.300 casos nuevos y 3.600 muertes en EE.UU. para el año $2007^{1,2}$. En Chile se describe como la segunda causa de muerte otorrinolaringológica, alcanzando una tasa de mortalidad de 0,8/100.000 habitantes ${ }^{3}$.

En más del $70 \%$ de los casos el diagnóstico se realiza en estadios tempranos, en donde las técnicas con conservación de órganos como radioterapia, cirugía parcial vía transcervical y microcirugía transoral láser describen similares resultados en cuanto a control locorregional, conservación de órganos y sobrevida global.

El uso del láser comienza a principios de los años 70 cuando el equipo de Strong y Jako realizan las primeras resecciones de lesiones laríngeas con láser CO2 lo cual fue evolucionando con el tiempo llegando a realizar entre otros procedimientos laringectomías parciales mediante este método $0^{5-7}$.

En la actualidad el láser CO2 alcanza su mayor rendimiento en los estadios tempranos de la enfermedad, con importantes ventajas respecto de los otros métodos conservadores ${ }^{8,9}$.

\section{OBJETIVOS}

\section{Objetivo general}

El objetivo general de este estudio es evaluar los resultados oncológicos de los pacientes con diagnóstico de cáncer de laringe que se trataron mediante microcirugía transoral láser en el Complejo Asistencial Barros Luco (CABL) desde enero de 2003 a diciembre de 2009.

\section{Objetivos específicos}

- Describir:

- Las características demográficas de los pacientes

- Presencia de factores de riesgo para esta neoplasia

- Ubicaciones del tumor primario

- Tamaño tumoral

- Presencia de metástasis a nodos cervicales y a distancia

- Tipo de histología

- Tratamiento a primario y cuello

- Complicaciones y tiempo de hospitalización

- Persistencia y recidiva de la enfermedad.

- Estimar la sobrevida global, control local inicial y control local final para dos años de seguimiento, según tamaño tumoral.

\section{MATERIAL Y MÉTODO}

Se diseñó un estudio retrospectivo descriptivo en donde se realizó una revisión de fichas de los pacientes con diagnóstico de cáncer de laringe y que fueron tratados mediante microcirugía transoral láser (CTL) en el Servicio de Otorrinolaringología del CABL entre enero de 2003 hasta diciembre de 2009.

Las variables incluidas fueron edad, sexo, consumo de alcohol y tabaquismo. Esta última se analizó de forma dicotómica si estaba presente 0 no el hábito y de forma continua expresando el consumo en forma de paquete/año.

Se consignó el tipo histológico de los tumores y se describió la ubicación anatómica del primario, tamaño tumoral, presencia de metástasis a nodos cervicales y a distancia según la clasificación TNM del American Joint Committee on Cancer (AJCC) ${ }^{10}$. 
Se describió el tipo de tratamiento que se realizó al primario consignando el tipo de cordectomía según la clasificación de la Sociedad Europea de Laringología (Figura 1) ${ }^{11,12}$.

Se estimó la sobrevida global que correspondió al tiempo transcurrido desde la intervención hasta el fallecimiento del paciente según datos actualizados a diciembre de 2009. Se entiende por control local inicial al tiempo desde la intervención hasta la aparición del primer evento local. El control local final corresponde al lapso desde la intervención hasta la obtención del control local mediante láser en un intervalo de tiempo determinado, sin necesidad de otra alternativa de rescate.

Los resultados se expresaron en números totales, proporciones, medianas y promedios con sus respectivas desviaciones estándar (DS). La sobrevida global, el control local inicial y final se expresaron en proporciones con un tiempo de seguimiento de 2 años. Los datos fueron tabulados y procesados en el programa estadístico SPSS versión 15.

\section{RESULTADOS}

Se logró reunir una muestra de 30 pacientes intervenidos con un promedio de edad de 58,9 años (DS $=12,2$ ) y un predominio del sexo masculino que alcanzó el $76,7 \%$ del total.

El $100 \%$ de los pacientes presentaba hábito tabáquico con una exposición promedio de 30 paquetes/año (DS =15) y el $60 \%$ refirió consumir alcohol.

En cuanto a la histología, todos los pacientes de esta serie correspondían a carcinoma escamoso.

La localización más frecuente fue la glótica con $80 \%$, seguido de la supraglótica en el $20 \%$ restante. Dos pacientes con compromiso glótico tenían extensión hacia subglotis.

A la mayoría de los pacientes se les diagnosticó un tamaño tumoral T2 seguido de T1 y T3 como se observa en la Figura 2.

Sólo 2 pacientes presentaron metástasis a ganglios cervicales. Uno correspondió a un $\mathrm{N} 1$ y el otro fue un N2b.

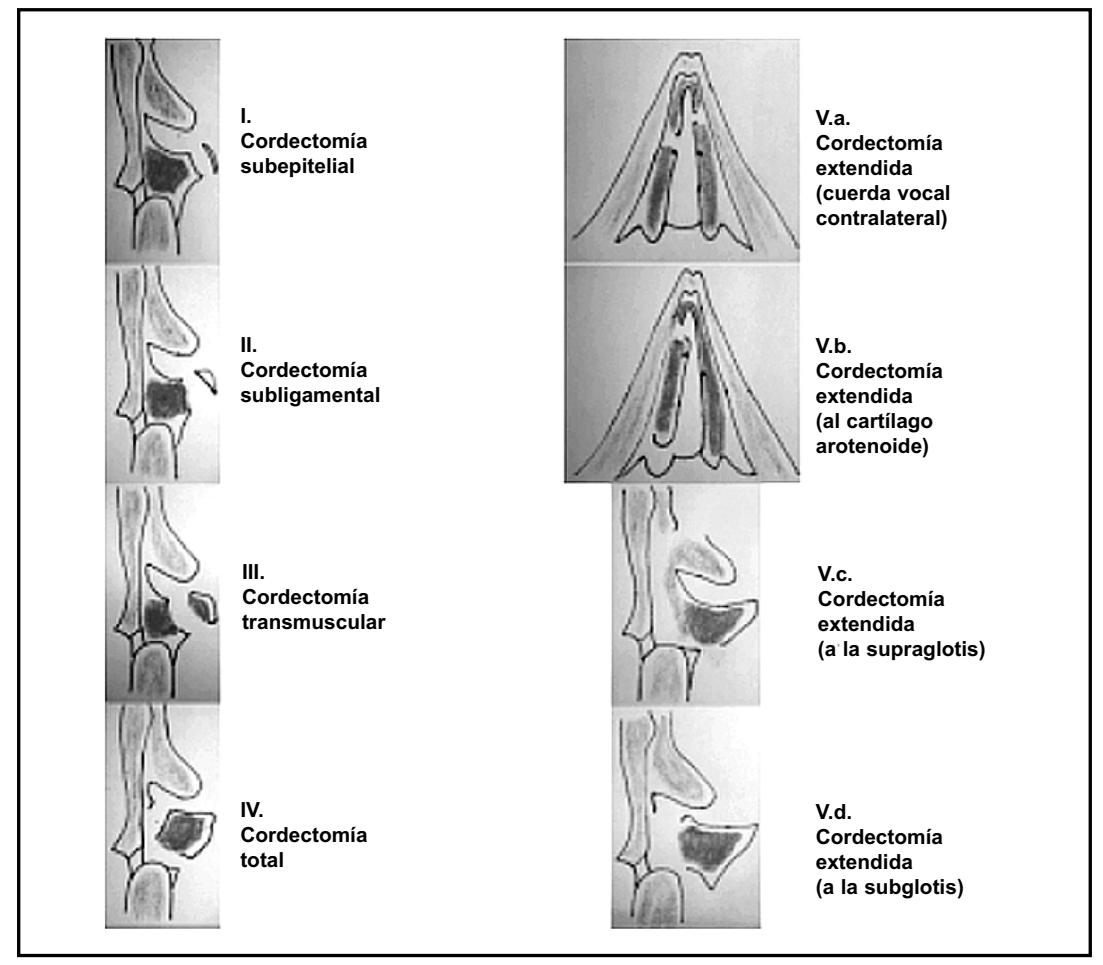

Figura 1. Tipo de cordectomía. Tomado de Zanolli y col. 


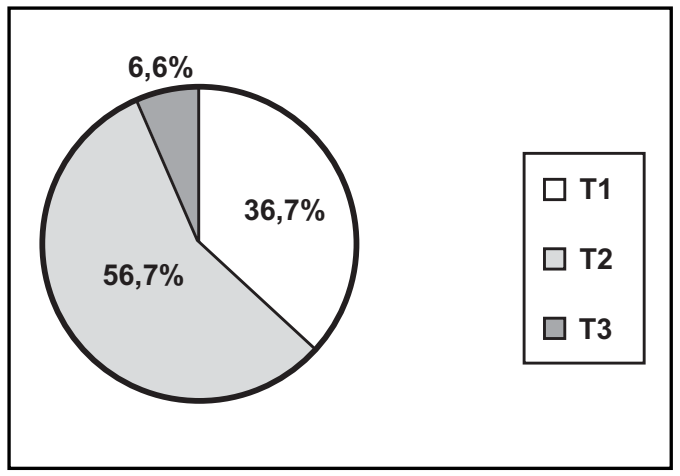

Figura 2. Distribución según tamaño tumoral $(n=30)$.

En cuanto al tratamiento, en la mayoría de los casos $(66,7 \%)$ se realizó una cordectomía tipo V y en 8 pacientes se requirió tratamiento del cuello por ser pacientes con compromiso supraglótico y subglótico (Tablas 1 y 2).

La complicación más frecuente fue la aspiración transitoria que afectó a tres pacientes con una media de duración de un día, cediendo de forma espontánea sin necesidad de tratamiento adicional. Tres pacientes presentaron sinequia de comisura anterior requiriendo traqueotomía transitoria en dos de ellos y posterior resolución quirúrgica de la sinequia.

Un paciente presentó un sangrado en el posoperatorio mediato y falleció por esta complicación.

El $70 \%$ de los pacientes fue dado de alta a las 24 horas poscirugía y el resto requirió mayor tiempo de hospitalización secundario a las complicaciones descritas, comorbilidad y necesidad de traqueotomía de seguridad.

Por sospecha de persistencia tumoral se reoperó a nueve pacientes y en seis de éstos se confirmó la presencia de tejido neoplásico. Siete pacientes requirieron una tercera operación y en dos una cuarta intervención (Tabla 3).

Para los cálculos de sobrevida global, control local inicial y final se incluyeron 19 pacientes que cumplieron el tiempo mínimo de seguimiento de 2 años. La sobrevida global (ajustada por muerte de causa neoplásica) fue de $100 \%$.

El control local inicial se alcanzó en el $77,8 \%$ y el control local final en $83,3 \%$ de los pacientes. En los casos T1 $(n=6)$ se logró una sobrevida global, control local inicial y final de $100 \%$. En los pacientes con tumores T2 $(n=11)$ se logró una sobrevida global de $100 \%$ y un control local inicial y final de $81,8 \%$. En los tumores T3 $(n=2)$ la sobrevida global fue de $100 \%$, no se logró control inicial y ambos pacientes requirieron una laringectomía total como alternativa de rescate (Tabla 4).

Tabla 1. Tratamiento de primario

\begin{tabular}{|lrr|}
\hline Tratamiento primario & $\%$ & $\mathrm{n}$ \\
\hline Cordectomía III & 3,3 & 1 \\
Cordectomía IV & 26,7 & 8 \\
Cordectomía V a & 50,0 & 15 \\
Cordectomía V c & 16,7 & 5 \\
Epiglotectomía & 3,3 & 1 \\
\hline Total & 100 & 30 \\
\hline
\end{tabular}

Tabla 2. Tratamiento de cuello

\begin{tabular}{|lrr|}
\hline Tratamiento primario & $\%$ & $\mathrm{n}$ \\
\hline Radioterapia & 13,3 & 4 \\
Vaciamiento cervical & 13,3 & 4 \\
Sin tratamiento & 73,3 & 22 \\
\hline Total & 100 & 30 \\
\hline
\end{tabular}


Tabla 3. Operaciones por sospecha de persistencia tumoral $(n=30)$

\begin{tabular}{|llcc|}
\hline Operaciones & $\mathrm{n}$ & $\%$ & Persistencia tumoral \\
\hline Segunda cirugía & 9 & 30,0 & $6(20 \%)$ \\
Tercera cirugía & 7 & 23,3 & $3(10 \%)$ \\
Cuarta cirugía & 2 & 6,7 & 0 \\
\hline
\end{tabular}

Tabla 4. Sobrevida global ajustada por muerte neoplásica. Control local inicial y final. Dos años de seguimiento. T1, T2 y T3

\begin{tabular}{|lcccr|}
\hline Tamaño tumoral & $\mathrm{n}$ & Sobrevida global & Control local inicial & Control local final \\
\hline T1 & 6 & $100 \%$ & $100 \%$ & $100 \%$ \\
T2 & 11 & $100 \%$ & $81,8 \%$ & $81,8 \%$ \\
T3 & 2 & $100 \%$ & $0 \%$ & $0 \%$ \\
\hline Total & 19 & $100 \%$ & $78,9 \%$ & $78,9 \%$ \\
\hline
\end{tabular}

\section{DISCUSIÓN}

En cuanto a la edad, sexo y presencia de factores de riesgo de los pacientes, los resultados de este trabajo son similares a lo descrito en el resto de la literatura ${ }^{13-15}$.

Al igual que en otras series publicadas, la localización con mayor número de casos fue la glótica seguida de la supraglótica, pero el tamaño tumoral T2 fue más frecuente que el T1, lo cual es contrario a lo descrito en la literatura ${ }^{16,17}$. Tomando en cuenta que en nuestro centro asistencial prácticamente todos los pacientes en estadios tempranos son tratados con cirugía transoral láser (CTL), esta cifra sería representativa de la real proporción de cáncer laríngeo precoz que está en control en nuestro Hospital. Una probable explicación de la mayor proporción de tumores T2 es la demora en la consulta y derivación de estos pacientes, que daría mayor tiempo de evolución desde el inicio de los síntomas.

La baja presencia de metástasis ganglionar se debe a que estamos tratando pacientes con tumores precoces y con gran proporción de localización glótica donde la diseminación neoplásica es infrecuente.

En cuanto al tipo de cordectomías se observa un predominio de la tipo $\mathrm{V}$ (Va y $\mathrm{Vc}$ ) lo cual se explica por la presencia de tumores T1 y T2 con sospecha de compromiso de comisura y de la necesidad de resecar la banda ventricular para obtener una mejor visión en los controles posoperatorios.

El manejo del cuello se rige por lo sugerido en las normas del National Comprehensive Cancer Network (NCCN) en que para tamaños tumorales iniciales se recomienda tratar cuando existe compromiso sub y supraglótico ${ }^{18}$.

Las complicaciones descritas en nuestro trabajo como aspiración y sinequia gótica son eventos esperables para este tipo de cirugía que se presentaron de forma similar a lo descrito en otras series $^{19-21}$.

El sangrado posoperatorio es otra complicación de este procedimiento, que al igual que un caso de nuestra serie, puede ser fatal, describiendo una mortalidad que fluctúa entre $0 \%$ y $0,3 \%$ según distintas publicaciones ${ }^{22,23}$. 
La CTL a diferencia de otras alternativas terapéuticas puede ser repetida si no se logra un control inicial de la cirugía. En la serie de pacientes del Hospital Clinic de Barcelona el $28,6 \%$ de los pacientes requirió una segunda intervención y el $14,3 \%$ más de dos cirugías, lo cual es semejante a nuestra experiencia salvo que mostramos una mayor proporción de pacientes que requieren más de dos intervenciones, lo cual se explica por la curva de aprendizaje necesaria para un óptimo control oncológico de los pacientes mediante este procedimiento ${ }^{24}$.

En cuanto a la sobrevida global, control local inicial y final, nuestros resultados son similares a lo expuesto por otras series. Si agrupamos los tumores T1 y T2 alcanzamos un control local y final de $88,2 \%$, muy semejante a otras experiencias como el grupo de Shvero y cols que con una casuística similar a la nuestra $(n=26)$ logró un control local inicial y final de $80 \%$ y $88 \%$, respectivamente ${ }^{25}$. Otras series con mayor número de casos muestran un control local inicial de $85 \%$ a $94 \%$ y un control local final de $89 \%$ a $98 \%{ }^{26-28}$.

Cabe señalar que estos estudios son con seguimientos de 5 años a diferencia de nuestra estimación que es a dos años, lo cual sobrestimaría nuestros resultados. Pero en nuestra serie, del total de pacientes que recidivaron, el $67 \%$ lo hizo antes del año de seguimiento y ninguno mostró recidivas luego de 18 meses.

\section{CONCLUSIONES}

Los resultados de control oncológico obtenidos en nuestro Servicio son comparables con los de la literatura internacional.

Los pacientes sometidos a CTL requieren tiempos reducidos de hospitalización y sus complicaciones son escasas y transitorias.

Los pacientes deben saber al inicio del tratamiento que es posible que tengan que someterse a más de un procedimiento quirúrgico.

Debido a la dinámica de las persistencias y recidivas tumorales en los pacientes que se ha realizado una CTL se deben seguir con nasofaringolaringoscopía y/o videofibroscopía mensualmente durante el primer año para luego distanciar los controles.

\section{BIBLIOGRAFÍA}

1. American Cancer Society. Cancer facts and figures. 2007. American Cancer Society; 2007. Disponible en:http://www.cancer.org/docroot/ STT/stt_0_2007.asp?sitearea=STT\&level=1.

2. Jemal A, Murray $T$, Ward $E$ y col. Cancer Statistics. Cancer J Clin 2005; 55(1): 10-30.

3. Béjar M, Cevo J, Romero M, IÑíguez R. Mortalidad nacional en otorrinolaringología. Rev Otorrinolaringología Cir Cabeza Cuello 2007; 67(1): 317.

4. BACK G, Sood S. The management of early laryngeal cancer: options for patients and therapists. Curr Opin Otolaryngol Head Neck Surg 2005; 13(2): 85-91.

5. Strong MS, JaKo GJ Y COL. The use of CO2 laser in otolaryngology: a progress report. Trans Am Acad Ophthalmol Otolaryngol 1976; 82 (5): 595-602.

6. Shapshay SM, Jako GJ. Laser surgery of the vocal cords; an experimental study with carbon dioxide lasers on dogs. Laryngoscope 1972; 82 (2): 2204-16.

7. Van Den Abbeele T, Trigla JM. Surgical removal of subglottic hemangiomas in children. Laryngoscope 1999; 109 (8): 1281-6.

8. Steiner W, Fierek 0, Ambrosch P, Hommerich CP, KRON M. Transoral laser microsurgery for squamous cell carcinoma of the base of the tongue. Arch Otolaryngol Head Neck Surg 2003; 129 (1): 36-43.

9. López A, Núñez F, Llorente Jl, Puente M, Aldama P, SuÁrez C. Cordectomías Láser: Resultados oncológicos y funcionales. Acta Otorrinolaringol Esp 2004; 55: 34-40.

10. Snehal G. Patel y Jatin P. Shah. TNM Staging of Cancers of the Head and Neck: Striving for Uniformity Among Diversity CA. Cancer J Clin 2005; 55 (4): 242-58.

11. Remacle M, Eckel he, Antonelli ar y col. Endoscopic cordectomy, a proposal for a classification by the Working Committee, European Laryngological Society. Eur Arch Otorhinolaryngol 2000; 257 (4): 227-31.

12. Zanoli L, Pinto R, Rahal M. Cáncer de laringe inicial cirugía transoral con láser. Review. Rev Otorrinolaringol Cir Cabeza Cuello 2007; 67 (1): 73-80. 
13. Pinto J, Nadal A, Mallofre $C$ y col. El efecto de la edad y de otros parámetros clínico-patológicos en el pronóstico del carcinoma escamoso de laringe: Utilidad y limitaciones. Informe preliminar. Rev Española Otorrinolaringol 2001; 61: 95-101.

14. Contreras J, Liebner A, Calabrese R, Plandiura J. Cáncer de laringe e hipofaringe: Evaluación de Ios años 1980 a 1988. Rev Otorrinolaringol Cir Cabeza Cuello 1989; 50: 25-33.

15. Koufman J, Burke, A. The etiology and pathogenesis of laryngeal carcinoma. Otolaryngologic Clinics 1997; 30 (1): 1-19.

16. Pukander J, Kerälä J, Mäkitie A, Hyrynkangas K, VIRTANIEMI J. Endoscopic laser surgery for laryngeal cancer. Eur Arch Otorhinolaryngol 2001; 258: 236-9.

17. Hoffman H, Porter K, Karnell L y COL. Laryngeal Cancer in the United States: Changes in Demographics, Patterns of Care, and Survival Laryngoscope. September 2006; 116: Supplement. National Comprehensive Cancer Network. Disponible en: http://www.nccn.org/index.asp.

18. Oeken J, Hänsch U, Thiel S, Bootz F. Swallowing function after endoscopic resection of supraglottic carcinoma with the carbon dioxide laser. Eur Arch Otorhinolaryngol 2001; 258: 250-4.

19. Steiner W, Аmbrosch P. Complications. In: Endoscopic Laser Surgery of the Upper Aerodigestive Tract. 2000, Georg Thieme Verlag, Stuttgart pp 112-113.
20. Eckel $H$, Schneider $C$, Jungehülsing M, Damm M, Schröder $U$, Vössing M. Potential role of transoral laser surgery for larynx carcinoma. Laser Surg Med 1998; 23: 79-86.

21. Vilaseca I, Bernal M, Blanch A, Moragas L. Complications in transoral CO2 laser surgery for carcinoma of the larynx and the hypopharynx. Head Neck 2003; 25 (5): 382-8.

22. Oliva M, Bartual J, Roquette J, Bartual J. Resultados del tratamiento del cáncer laríngeo supraglótico mediante cirugía endoscópica con láser de C02. Acta Otorrinolaringol Esp 2003; 54: $569-74$

23. Bermal M, Blanch J, Vilaseca I, Steiner W, Ambrosch P. Cirugía con Láser CO2 en la vía aerodigestiva superior. Almirall $20041^{a}$ Edición.

24. Shvero J, Koren R, Zohar L y COL. Laser surgery for the treatment of glottic carcinomas. $A m \mathrm{~J}$ Otolaryngol 2003; 24 (1): 28-33.

25. Eckel $H$, Thumfart W, Jungehulsing $M$ y col. Transoral laser surgery for early glottic carcinoma. Eur Arch Otorhinolaryngol 2000; 257 (4): 221-6.

26. Peretti G, Piazza C, Bolzoni A y col. Analysis of recurrences in $322 \mathrm{Tis}, \mathrm{T} 1$, or $\mathrm{T} 2$ glottic carcinomas treated by carbon dioxide laser. Ann Otol Rhinol Laryngol 2004; 113 (11): 853-8.

27. Steiner W. Results of curative laser microsurgery of laryngeal carcinomas. Am J Otolaryngol 1993; 14 (2): 116-21.

Dirección: Dr. Rodrigo Arregui V.

Gran Avenida José Miguel Carrera 3204, San Miguel. Santiago

E mail: rarregui@gmail.com 